\title{
GEOMETRY OF INTEGRABLE LATTICE EQUATIONS AND THEIR REDUCTIONS
}

\author{
MATTHEW NOLAN
}

(Received 3 March 2019; first published online 17 May 2019)

2010 Mathematics subject classification: primary 14J17; secondary 14J17, 39A06, 39A14.

Keywords and phrases: singularities, blow up, linear equations, $q$-difference equations, partial difference equations, lattice equations, Painlevé equation.

Modern research into discrete integrable systems has provided new insights into a wide variety of fields, including generalisations of special functions, orthogonal polynomials and dynamical systems theory. In this thesis, we extend one of the most productive insights in this area to higher dimensions. In particular, we show how to apply ideas from resolution of singularities and birational geometry to discrete systems in higher dimensions.

The most widely studied setting for these ideas lies in spaces of dimension two. By blowing up at certain points to resolve singularities found in maps on surfaces, new surfaces are constructed on which the map becomes an isomorphism, a so-called space of initial conditions. This has led to new developments in the field, including the discovery of new examples of integrable maps by Sakai [3] with solutions that have unexpectedly rich properties.

On the other hand, this geometric approach has never been applied to integrable partial difference equations (often called lattice equations), which share other properties with the maps in dimension two. In this thesis, we overcome this gap.

In particular, we examine spaces of initial conditions for integrable lattice equations, which are members of the equations classified by Adler et al. [1], known as ABS equations. By explicitly calculating the induced map on their resolved initial value spaces, we find transformations to new lattice equations, and hence find novel reductions to discrete Painlevé equations. We also show that an equation arising from the geometry of ABS equations is satisfied by the coefficients of a cluster algebra associated with a form of the discrete modified Korteweg-de Vries equation. This work can be found in [2].

Thesis submitted to The University of Sydney in April 2017; degree approved on 17 September 2018; supervisor: Nalini Joshi.

(C) 2019 Australian Mathematical Publishing Association Inc. 


\section{References}

[1] V. E. Adler, A. I. Bobenko and Y. B. Suris, 'Classification of integrable equations on quad-graphs. The consistency approach', Commun. Math. Phys. 233(3) (2003), 513-543.

[2] N. Joshi, S. Lobb and M. Nolan, 'Constructing initial value spaces of lattice equations', Preprint, 2018, arXiv:1807.06162.

[3] H. Sakai, 'Rational surfaces associated with affine root systems and geometry of the Painlevé equations', Commun. Math. Phys. 220(1) (2001), 165-229.

MATTHEW NOLAN, School of Mathematics and Statistics, University of Sydney, Camperdown, NSW 2006, Australia

e-mail: m.nolan@maths.usyd.edu.au 